\title{
LIQUID CRYSTAL V.A.N. TILT BIAS BY SURFACE RELIEF PATTERNING
}

\author{
Mark C. Gardner, Sally E. Day, F. Anibal Fernandez \\ Department of Electronic \& Electrical Engineering \\ University College London, Torrington Place, London WC1E 7JE \\ Tel: +44(0)2076793195 Fax: +44(0)207388925 E-mail: m.gardner@ee.ucl.ac.uk
}

\section{ABSTRACT}

Liquid Crystal Displays require controlled alignment of the liquid crystal molecular director at its confining surfaces. These surfaces may be coated glass or in the case of 'Liquid Crystal On Silicon' (LCOS) technology, a silicon backplane. In the case of Vertically Aligned Nematic (VAN) cells an initially vertical orientation is used and from this the director may tilt in any direction. Some means is required to bias the tilt in a consistent direction to avoid the occurrence of differently oriented domains. For VAN cells one tilt bias method is oblique deposition of silicon oxide. An alternative method which eliminates concerns over consistency of deposition angle over a large substrate area is the use of surface relief structures to induce tilt bias. This is attractive for LCOS devices. Liquid crystal modelling tools [1] have been used to simulate the effects of rectangular and triangular shaped 'bumps' and 'dips' protruding from and extending into the LC's enclosing surfaces respectively. The director orientation and optical transmission of the LC pixels biased in this way are examined as a function of time during the switching cycle and spatially across the pixel to show that the combination provides controllable tilt bias.

\section{INTRODUCTION}

For high information content displays, and in particular projection systems, high contrast ratio is a prime requirement. One means of achieving superior image contrast over the commonly used Twisted Nematic (TN) liquid crystal (LC) configuration is to use the Vertically Aligned Nematic (VAN) mode. This configuration can ideally provide total dark state extinction when placed between crossed polarisers.

The VAN cell employs a liquid crystal material having a negative dielectric anisotropy, $\Delta \varepsilon$, and positive optical anisotropy, $\Delta \mathrm{n}$. The material is confined between substrates which impose homeotropic alignment, i.e. with the liquid crystal director (the mean molecular orientation over a microscopic region) aligned normal to the substrates. In the OFF state, normally incident light encounters the 'ordinary' refractive index, $\mathrm{n}_{\mathrm{o}}$ of the liquid crystal material, and so passes through with no change in its polarization. Placing crossed polarisers around the cell blocks the light, giving a dark state. The quality of this dark state is limited only by the polariser performance and the uniformity of LC alignment and is therefore generally very good. When in the $O N$ state, the applied field reorients the LC director through the negative $\Delta \varepsilon$, along the plane of the cell. In this condition, the cell acts as a birefringent plate. Thus, if the thickness of the cell satisfies the condition: $\Delta \mathrm{n} . \mathrm{d} / \lambda=0.5$ the cell can be considered a half-wave plate. ( $\Delta \mathrm{n}$ here represents the mean effective optical 
anisotropy since the director alignment near the substrates remains close to homeotropic.)

If the cell is placed between crossed polarisers so that the axis of the half-wave plate bisects the enclosing polariser angles, the $O N$ liquid crystal layer will rotate the incident polarisation to emerge from the second polariser. If operated in a reflective mode, for example using Liquid Crystal On Silicon (LCOS) technology, the LC layer thickness can be reduced by half while keeping the same total optical path-length. A reflective configuration has the benefit of reducing the switching speed of the device since switching times are proportional to the square of the cell gap. With switching times in the region of $2 \mathrm{~ms}$, LCOS sequential colour displays become realisable.

When switching from the $O F F$ to the $O N$ state, some means must be employed to bias the tilt direction of the $O N$ state director relative to the polarisers. This can be provided by a small pre-tilt away from homeotropic alignment at the substrates. This initial tilt bias will then allow uniform switching without the formation of uncontrolled differently oriented domains. The tilt angle in the OFF state should be small to ensure high contrast, and of high uniformity to ensure similar switching times and required alignment relative to the polarisers in all parts of the display. The nearhomeotropic (often called tilted-homeotropic) alignment is usually applied using either oblique deposition of oxides of Silicon, $\mathrm{SiO}_{\mathrm{x}}$ [2] or by directional rubbing [3]. When oblique $\mathrm{SiO}_{\mathrm{x}}$ deposition is used, the angle between director and deposited layer depends not only on deposition angle, but also on the LC material. Lu et al [4] found that for an angle of deposition between $30^{\circ}$ and $60^{\circ}$, the director for an LC with positive $\Delta \varepsilon$ is homogeneous (parallel with the substrate), while for a negative $\Delta \varepsilon$, it is nearly homeotropic with a tilt between $0^{\circ}$ and $3^{\circ}$. This suggests that the angle made between $\mathrm{SiO}_{\mathrm{x}}$ and director may depend partly on the chemistry of the LC molecule.

While the VAN cell provides good contrast, and a good viewing angle in the OFF state, a good viewing angle in the $O N$ state requires division of pixels into multiple domains, where neighbouring domains switch towards opposing directions. The brushing and oblique deposition methods of uniform alignment do not permit the fabrication of small well-defined domains. As an alternative, relief features in the substrates can be used to provide a small tilt bias sufficient to provide multiple domains of orientation within a single pixel. Tilt bias using relief features also avoids the requirements of controlled $\mathrm{SiO}_{\mathrm{x}}$ deposition angle and the high curing temperatures of spin-coated polyimide. Such multiple domain configurations can be applied to direct view displays where a wide viewing angle is desirable. 
VAN technology is important for projection displays because of the need to maintain a high contrast and a bright $O N$ state, while the requirement for wide viewing angle is not so critical. Control of the LC orientation in the $O N$ state is therefore important in order to maintain the brightest $O N$ state, rather than viewing angle. This means that for projection displays it is not a requirement to have multiple domains, only that the orientation is well controlled. In this paper we present 2-dimensional simulations of tilt bias induced by surface relief features on the lower substrate of a VAN liquid crystal cell.

The projection LCOS display has reflective pixel electrodes and so boundaries to the visible pixel area are defined by the electrodes themselves rather than an opaque mask layer placed on top of the electrodes. In contrast to the direct view display, each single tilt domain can extend over the area of one full pixel, and any disclinations between the domains should be localised to the inter-pixel gaps (non-reflective areas). Furthermore, a normally black operating mode means that disclinations appearing in the $O N$ state will show as dark regions between the pixels, thus contrast is not strongly affected.

\section{DOMAIN INDUCING STRUCTURES}

A number of methods have been put forward for inducing tilt domains in VAN cells using dielectric protrusions and/or patterned electrodes. Takeda et al introduced the "Automatic Domain Formation" (ADF) mode [5]. Regularly spaced triangular protrusions are placed alternately on the top and bottom substrates. Arranging the protrusions in the form of chevrons allowed the creation of 4-domain pixels. Registration of upper and lower substrates is necessary for effective operation. Tanaka et al, noted that field effects around the periphery of pixels caused unwanted disclinations [6]. These were overcome by the introduction of 'wings' to the protrusions. The same authors then put these edge field effects to good use by replacing the lower protrusions of Takeda et al with slits in the lower electrodes to form 'virtual protrusions'. Yoshida et al used dielectric layers on the substrates to engineer the field lines of an in-plane switching VAN cell [7]. The layers allowed the production of multiple domains by controlled oblique fields. Kwag et al introduced the "Enhanced Vertical Alignment" (EVA) mode [8]. Of these methods, that proposed by Kwag et al is perhaps of particular interest for projection LCOS applications. They suggest a series of dielectric protrusions into the LC layer where alternate protrusions either penetrate the pixel electrode or are covered by it. The asymmetric field from the patterned electrodes tends to tilt the director away from the non-conducting protrusions, and towards the conducting ones. Any disclination between the regions of different tilt are then localised over the protrusions. Thus the two aims of controlled tilt bias and localised disclinations are met.

In our method for projection LCOS, a protrusion in the centre of an electrode would be unacceptable as it would distort the plane mirror surface and severely degrade the normal incidence reflectivity. Instead of the electrode covered protrusion we propose the use of a recess or 'dip' into the silicon side substrate. Recall that in contrast to the direct view cell, we do not require the division of individual pixels into domains, but seek controlled tilt bias allowing a single domain over the pixel area. Domain inducing features can therefore be placed over the inter-pixel gaps. In the following then, two feature types are investigated comprising small protrusions into the LC layer 
and small recesses into the substrate. We refer to these features as bumps and dips respectively. The two types of feature can be used in combination to provide a uniform tilt bias over each pixel. The dips provide a similar function to the electrode covered protrusions in Kwag's EVA method. A regular pattern of alternate 'bumps' and 'dips' can then be used to induce single tilt domains over each pixel. Figure 1 gives a simplified illustration of the induced tilt bias.

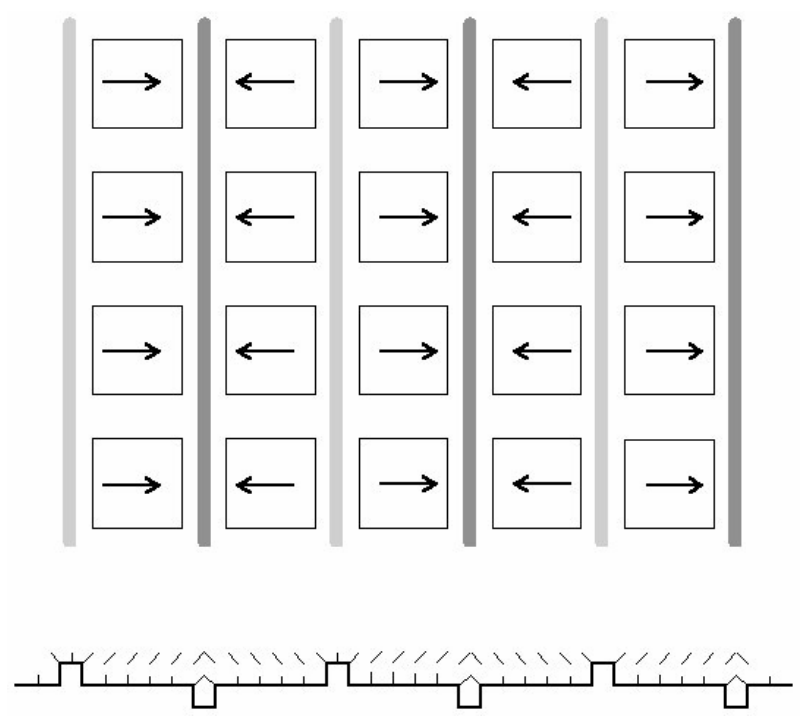

Fig. 1 Pixel array showing effect on director of alternating rows of bumps and dips (plan \& section)

\section{$4 \quad$ 2D SIMULATION IN THE VICINITY OF FEATURES}

The effect of each feature type will be examined individually and then in combination to represent a pixel array. In all the following computer simulations local homeotropic alignment is assumed, i.e. the LC director is assumed to be anchored normally to the local surface of the features as if no surface treatments or special deposition techniques are used. Rectangular and triangular shapes were considered in these simulations. The relaxed OFF state director configuration around each form of bump and dip are illustrated below Fig. 2.

It is clear that the bumps strongly influence the surrounding director field, whereas the dips of the same dimensions have only a localised effect at their corners. This might be expected since the bump protrudes into the liquid crystal regions further from the rigid anchoring at the substrates while the dips are somewhat hidden.

However, the local distortion to the director field due to anchoring is not the only factor determining the direction of tilt during switching. The presence of the features modifies the electric field lines around the underlying pixel electrodes. The angle between electric field lines and LC director have a strong effect on the direction in which the director will reorient [ 7,8,9]. This is especially important in the dip case where the shape of the electric field can oppose and override the effect of the surface feature anchoring. The effect is best visualized by considering the equipotential lines in figure 3. For an LC with negative anisotropy, the director will tend to align with the equipotential lines. 


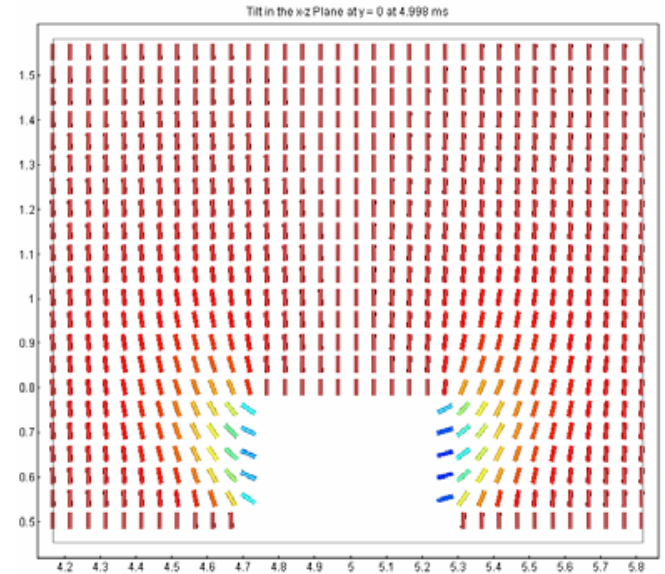

(a)

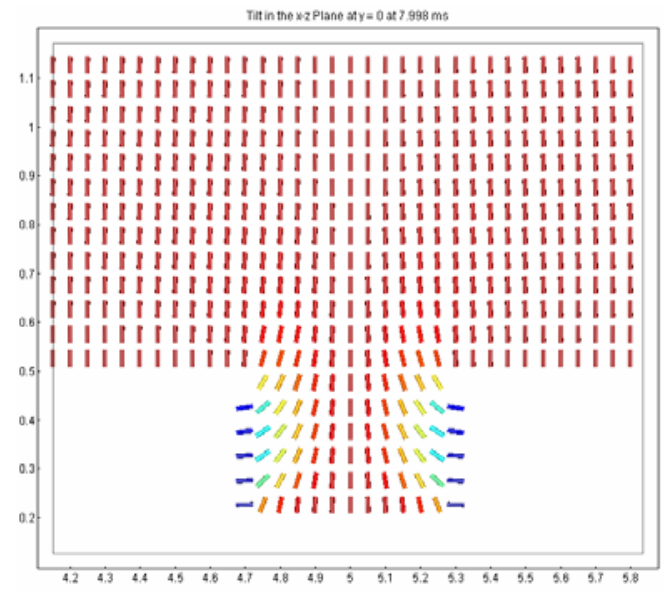

(c)

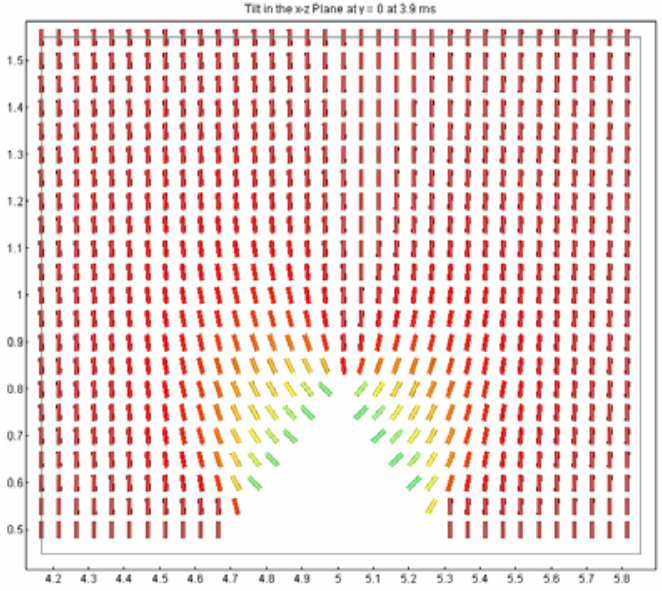

(b)

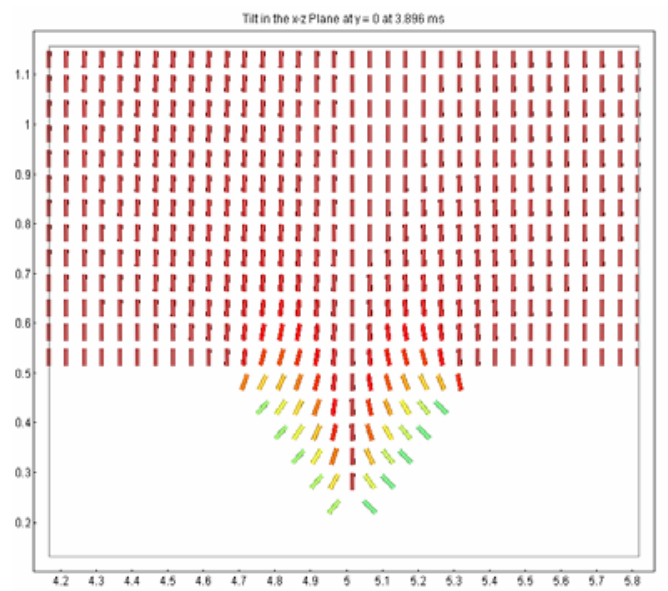

(d)

Fig. 2 The director field in the vicinity of a selection of relief feature types.

a) rectangular bump, b) triangular bump c) rectangular dip d) triangular dip.

Thus an initially vertical director will re-orientate in the direction making the smallest angle with the equipotential line. Equipotential patterns are illustrated below for small differences in the dip and electrode configurations immediately after application to the un-switched cell. It is seen below that for a triangular dip there is only a sharp dip in the equipotentials. Consider the right hand side of the plots in figure 3. Near the sharp dip in the equipotentials around features TD2 and TD4 the director would tend to reorient clockwise, which is in opposition to the condition we are aiming to achieve from a dip feature. Similarly, rectangular dip SD2, there is a small dip in the equipotential lines. For SD4 however, the small region where the equipotential dip is surrounded by a wider region of opposite slope which favours the required tilt bias direction (anti-clockwise). Furthermore, these outer slopes coincide with the area where the LC director is tilted due to the anchoring on the dip feature. 


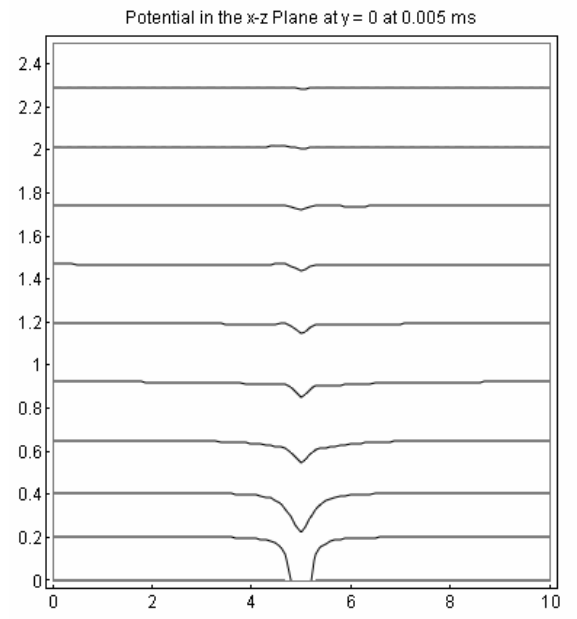

(a)

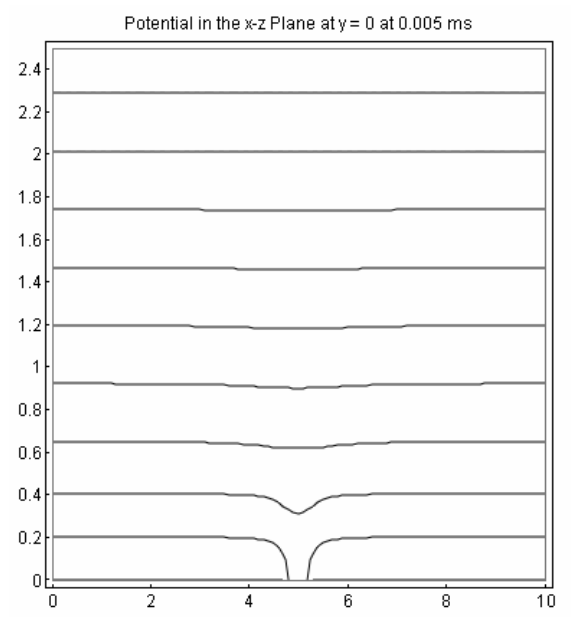

(c)

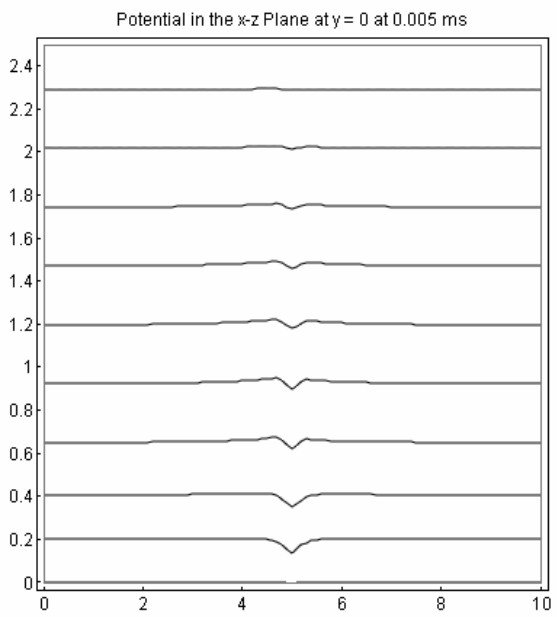

(b)

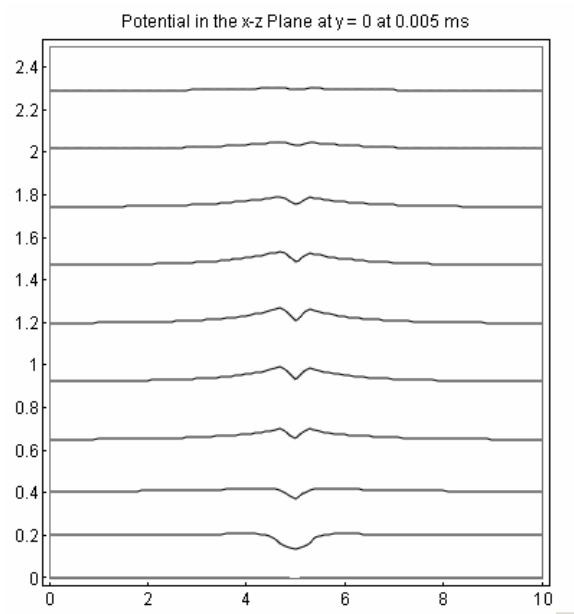

(d)

Fig. 3 Equipotentials resulting from 4 feature configurations

a) Triangular dip with $0.6 \mu \mathrm{m}$ base, $0.3 \mu \mathrm{m}$ depth, electrodes $0.2 \mu \mathrm{m}$ below dip floor, $0.6 \mu \mathrm{m}$ pixel gap (type TD2)

b) Triangular dip with $0.6 \mu \mathrm{m}$ base, $0.3 \mu \mathrm{m}$ depth, electrodes $0.2 \mu \mathrm{m}$ below dip floor, $0.1 \mu \mathrm{m}$ pixel gap (type TD4)

c) Rectangular dip with $0.6 \mu \mathrm{m}$ width, $0.3 \mu \mathrm{m}$ depth, electrodes $0.2 \mu \mathrm{m}$ below dip floor, $0.6 \mu \mathrm{m}$ pixel gap (type SD2)

d) Rectangular dip with $0.6 \mu \mathrm{m}$ base, $0.3 \mu \mathrm{m}$ depth, electrodes $0.2 \mu \mathrm{m}$ below dip floor, $0.1 \mu \mathrm{m}$ pixel gap (type SD4)

So, it is seen that the effectiveness of these surface features depends not only on their shape but also on the relative location of the electrodes beneath them. The triangular and rectangular dips show relatively small relaxed tilt bias compared to the bump features. When a field is applied, a triangular dip located over the electrode gap accentuates the trough in equipotentials expected over any electrode gap which tends to reorient the director in the wrong direction. A rectangular dip which is wider than the electrode gap allows the field above the electrode surfaces to penetrate further into the LC layer and so produces a favourable slope to the equipotentials encouraging tilt bias towards the feature. The region of favourable slope increases with the ratio between dip-electrode overlap to electrode gap. Hence, a small electrode gap is desirable beneath a rectangular dip feature. Conversely, a large electrode gap is 
desirable beneath a bump feature to produce a deep through in the equipotentials, encouraging tilt bias away from the feature comparable to the effect seen in electrode gaps in Kwag's paper [8].

The two types of feature are employed in pairs so that each pixel has a bump on one side and a dip on the other. The bumps and dips work in unison to induce a uniform direction of tilt over the pixel. Alternate pixels will have alternate directions of tilt bias as suggested previously, (Fig. 1). In consideration of the observations concerning slope and electrode separation, rectangular bumps (type SB2), and dips (type SD4) were chosen to demonstrate the two types of feature working in pairs. The operation of the bump-dip pair is illustrated in figure 4 where the director movement due to the features and applied field can be seen. Figure 4 a) shows the director field in the relaxed $O F F$ state. The simulations used to generate these results employed periodic boundary conditions so that the interval of two pixels separated by alternative bumps and dips in the figure is, in effect, repeated indefinitely to approximate to a large array of pixels on a display device [1]. Figure $4 \mathrm{~b}$ ) shows the cell during switching. It is clear that the direction of tilt is uniform over each pixel with disclinations between tilt domains located over the pixel gaps. The bump is seen to be the main 'seed' for the direction of tilt bias while the dip serves mainly to lock the disclination over the pixel gap. Figure $4 \mathrm{c}$ ) shows the cell in an almost fully switched state. The directors at the pixel centres are seen to be almost horizontal, with higher tilts only found close to the relief features.

\section{OPTICAL TRANSMISSION ALONG A SECTION}

Optical transmission for the cell considered above for the same three stages of switching are shown in figure 5 . In figure 5 a), the dark state intensity is even over the pixel except near to the features. In fact, for the dip, the higher intensity region exists only within the walls of the dip itself. In figure $5 \mathrm{~b}$ ), shows how the bright region sweeps out from the bump towards the dip, while the disclinations are locked over the inter-pixel gaps. Finally, in figure 5 c) the cell is strongly switched and dark areas are located only between pixels.

Note that the shapes used in these simulations were idealised forms with sharp corners and flat edges. In practice the capabilities of the fabrication stages will govern both the size and shapes of features. Similarly there are other practical limitations to the electrode shape and dimensions which will limit the inter-pixel gap such as the small separation between pixels beneath the dip feature. The differences in electric field patterns resulting from small changes to the dips and electrodes in figure 3 suggest that good control of shapes and dimensions during fabrication is necessary to ensure effective control of tilt bias and switching.

For ease of simulation, the ratio of pixels size $(8.25 \mu \mathrm{m})$ to pixel gap $(0.5 \mu \mathrm{m} \&$ $0.1 \mu \mathrm{m})$ used was rather less than would be expected in practice. So in a real cell, the dark areas between pixels would be expected to occupy a much smaller area of the total display. However, the way in which the switching sweeps out from the bump features is likely to cause slower switching as pixel size is increased. 
Potential in the $x-z$ Plane at $y=0$ at $13.896 \mathrm{~ms}$

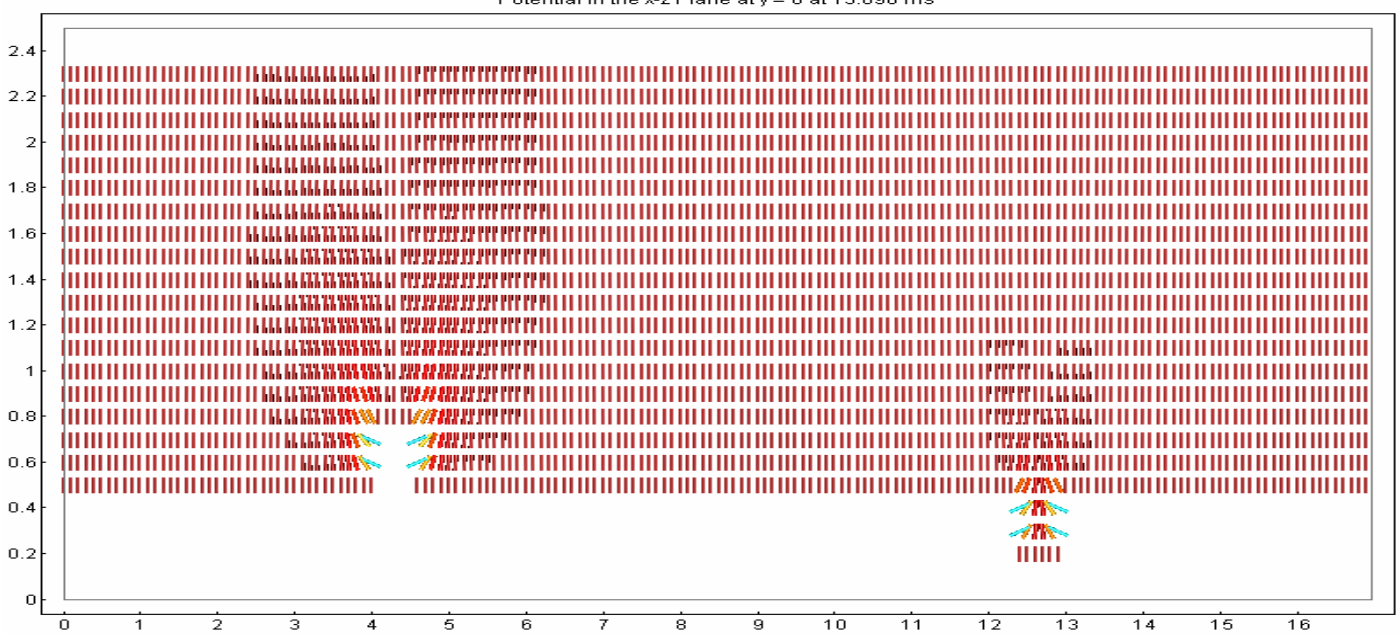

(a)

Potential in the $x-z$ Plane at $y=0$ at $17.496 \mathrm{~ms}$

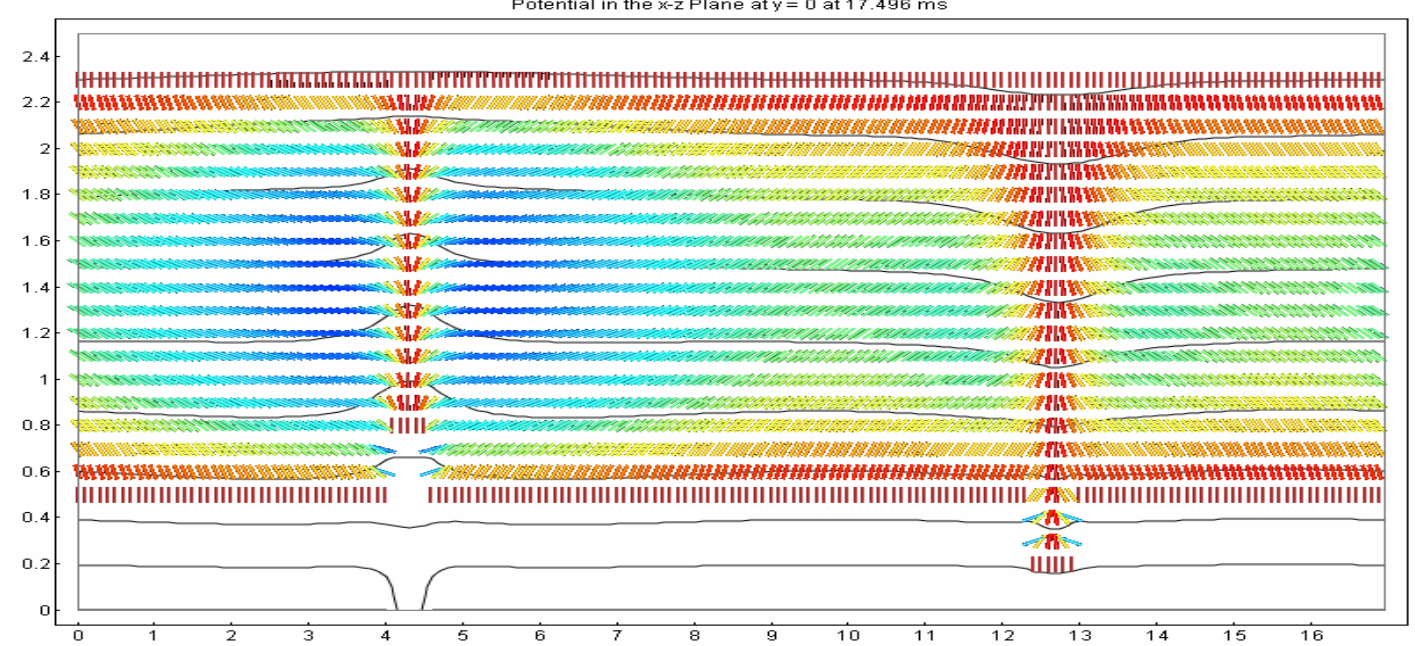

(b)

Potential in the $x-z$ Plane at $y=0$ at $21.996 \mathrm{~ms}$

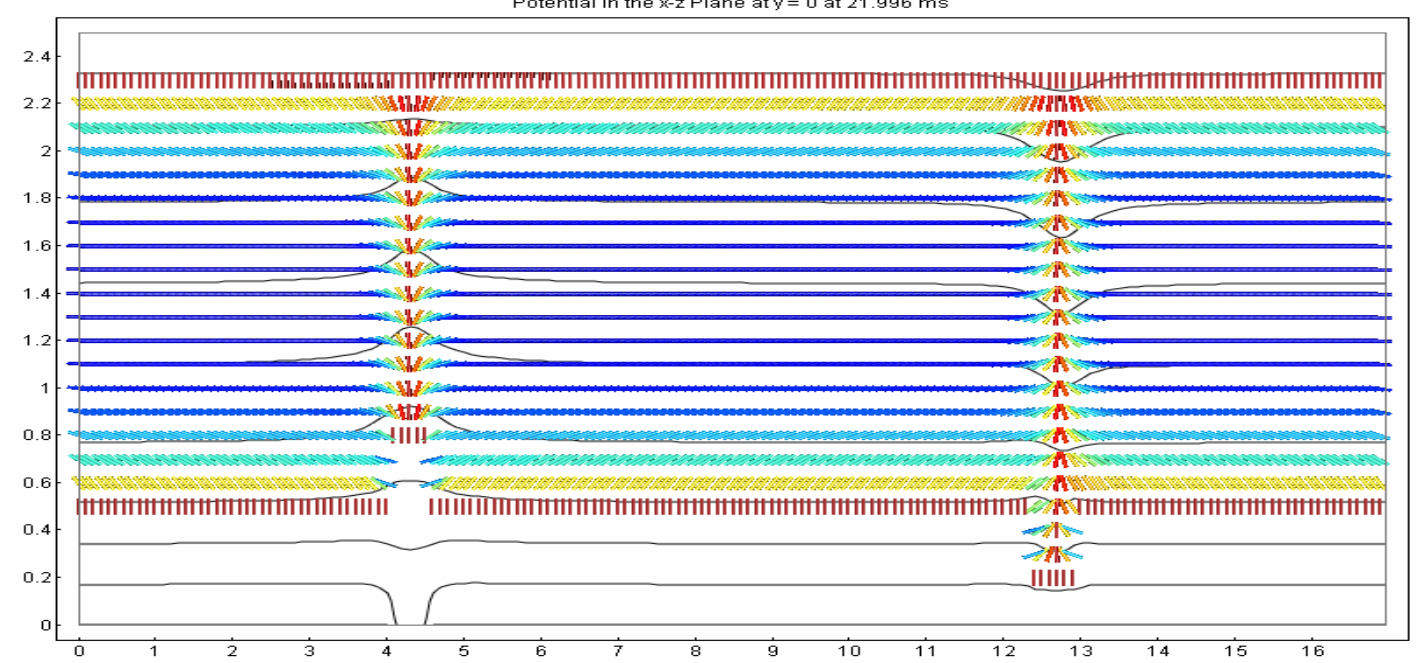

(c)

Fig. 4 Tilt bias by the combined effect of 'bumps' and 'dips'; a) un-switched b) switching c) switching 


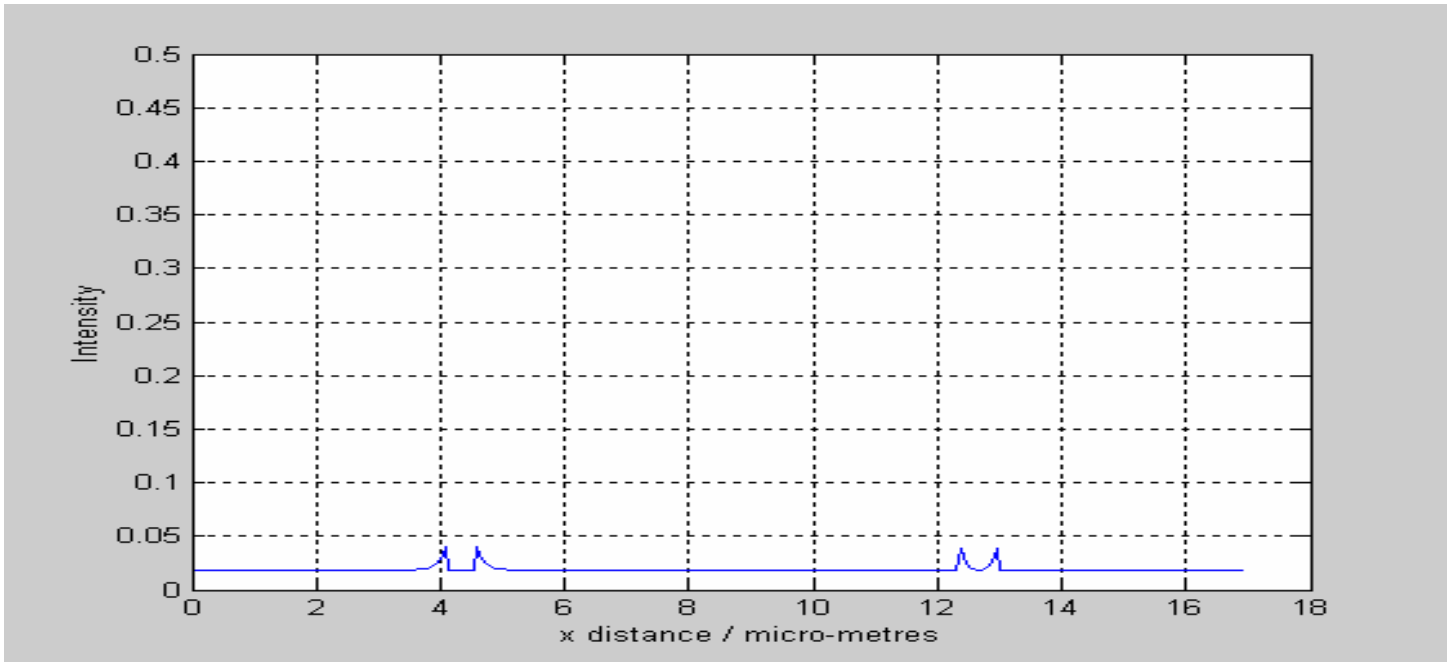

(a)

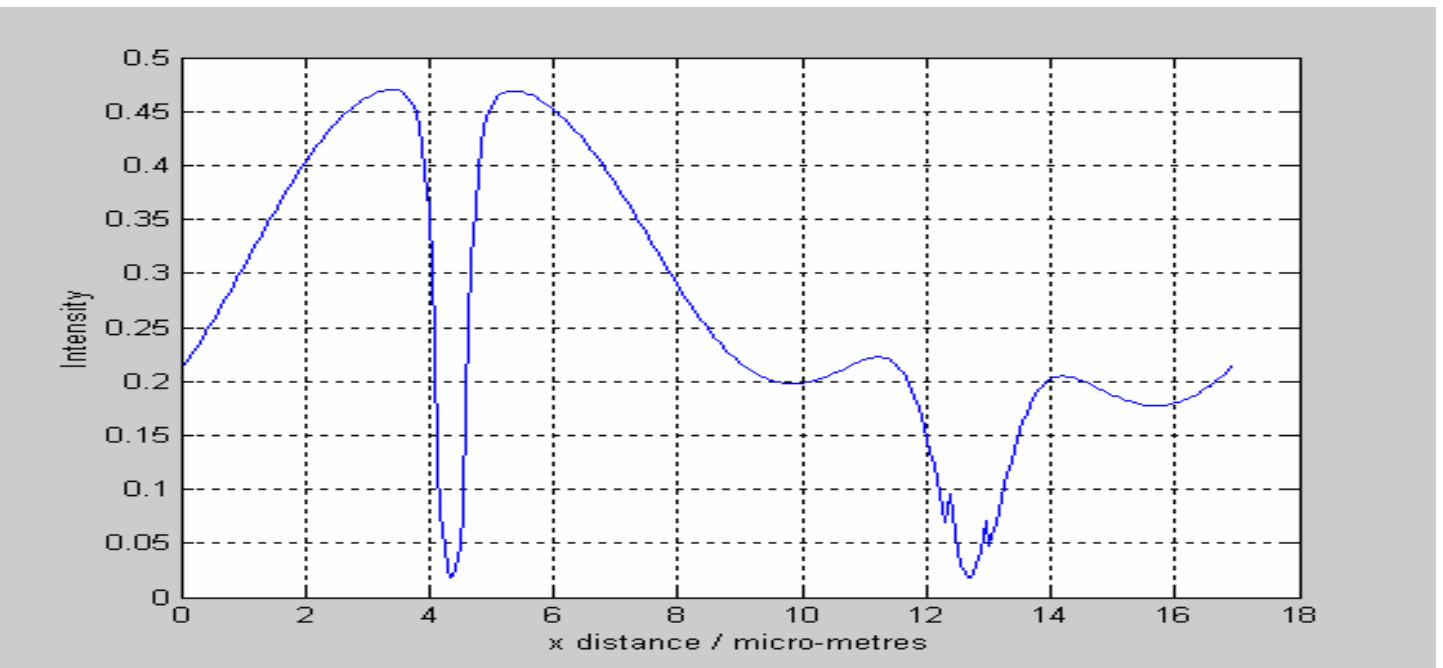

(b)

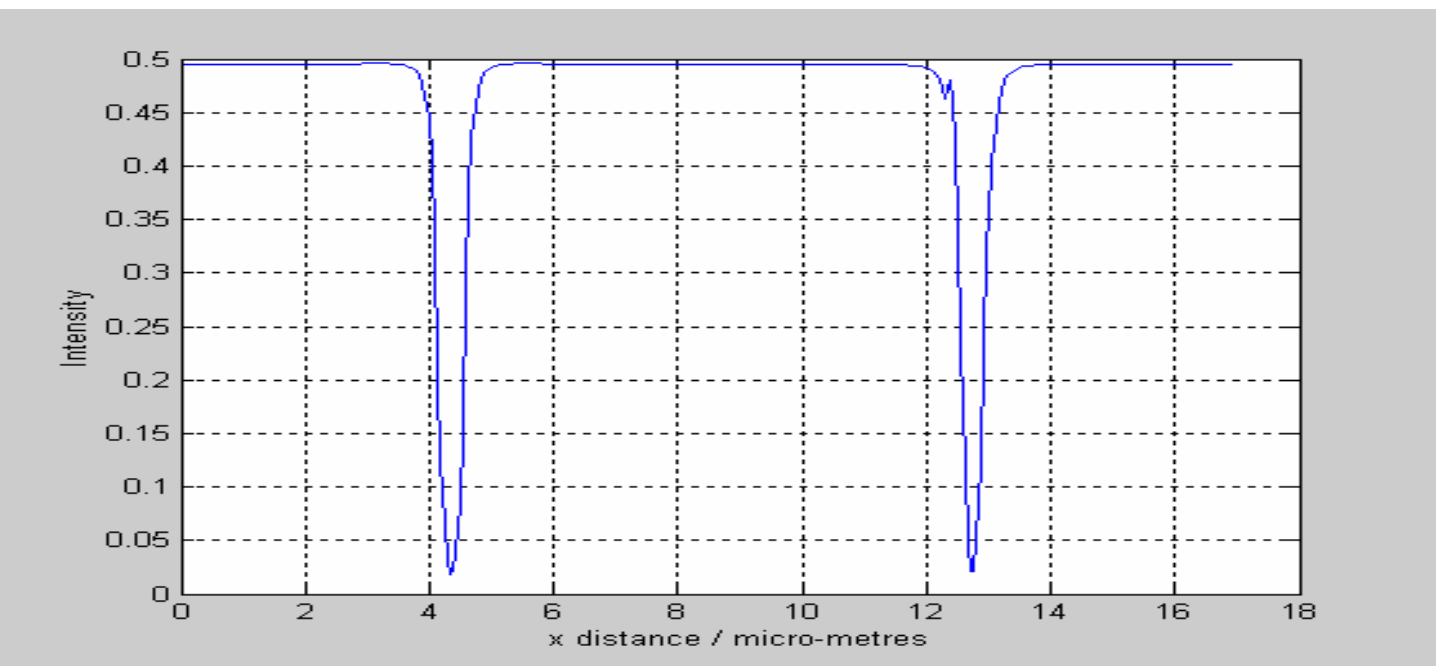

(c)

Fig. 5 Tilt bias by the combined effect of 'bumps' and 'dips'; a) un-switched b) switching c) switched 


\section{$6 \quad$ CONCLUSIONS}

A method of employing protrusions and recesses in one substrate of a liquid crystal cell has been proposed to produce the tilt bias required in the Vertical Aligned Nematic mode. The method is suitable for projection displays where high contrast and high brightness are more important than a wide viewing angle. The method provides some advantages over the conventional techniques of brushing and oblique deposition of $\mathrm{SiO}_{\mathrm{x}}$. The bumps and dips used in this method can be fabricated by photolithography. There is no requirement for extra electrodes and the upper substrate need only be a common plane electrode, hence there are no registration difficulties between upper and lower substrates.

In the OFF condition, the liquid crystal director is very nearly vertical, (except for the small region close to the bump and dip surfaces) ensuring a high quality dark state. In the $O N$ condition, the disclination between differently oriented domains is located over the inter-pixel gap and no artefacts should be seen within pixels so that overall brightness is not affected.

The simulations have been performed on geometric triangular and rectangular shapes and both shapes show uniform switching. It has been shown that the combination of the shape and size of the surface relief structure and the gap in the electrodes below these must be chosen appropriately. While the bumps and dips used in these simulations are of similar size, it was found that the bumps have a greater impact on the surrounding director than the dips. The detailed structures achievable during fabrication can be included in the methods described above to finalise the exact structures required in a practical device

\section{Acknowledgements}

This work was carried out with support from the European Commission under the Framework 5 program, project LCOS4LCOS, N IST-2001-34591 


\section{REFERENCES}

1 J.B. Davies, S.E. Day, F. Di Pasquale and F.A. Fernández, "Finite element modelling in 2-D of nematic liquid crystal structures" Electron. Letts., vol. 32, Nº 6, pp. 582-583, March 1996

2 Janning JL "Thin film surface orientation for liquid crystals" Appl. Phys. Lett. Vol. 21 p173 (1972)

3 J. Cognard, "Alignment of nematic liquid crystals and their mixtures," Mol. Cryst. Liq. Cryst. Suppl. Vol.1, p1 1982

$4 \quad$ Lu M. Yang KH, Nakasoki T, Chey SJ, "Homeotropic alignment by single oblique evaporation of $\mathrm{SiO}_{2}$ and its application to high resolution microdisplays"

SID Tech. Digest 31, p446 (2000)

5 Takeda S, Kataoka T, Sasaki T, Chida H, Tsuda K, Ohmuro K, Sasabayashi T, Koike T, Okamoto K

"A super-high image quality multi-domain vertical alignment LCD by new rubbing-less technology"

SID Tech. Digest p1077 (1998)

6 Tanaka Y,Taniguchi Y, Sasaki T, Takeda A, Koibe Y, Okemoto K "A new design to improve performance and simplify the manufacturing process of high-quality MVA TFT-LCD panels" Late-news paper SID Tech. Digest p206 (1999)

7 Yoshida H, Nakanishi Y, Sasabayashi T, Tasaka Y, Okamoto K, Inoue Y, Sukenori H, Fujikawa T

"Fast-switching LCD with multi-domain vertical alignment driven by an oblique electric field"

SID Tech Digest p334 (2000)

8 Kwag JO, Shin KC, Kim JS, Kim SG, Kim SS

"Implementationof a new wide viewing angle mode for TFT -LCDs" SID Tech. Digest p256 (2000)

$9 \quad$ Koma N, Baba Y, Matsuoka K "No-rub multi-domain TFT-LCD using surrounding-electrode method" SID Tech. Digest p869 (1995) 